Olitzki, A. L. (1954). J. gen. Microbiol. 11, 160-174.

\title{
Hydrogen Sulphide Production by Non-multiplying Organisms and its Inhibition by Antibiotics
}

\author{
By A. L. OLITZKI \\ Department of Bacteriology, Hebrew University-Hadassah Medical School, \\ Jerusalem, Israel
}

SUMMARY: Hydrogen sulphide production by washed bacterial suspensions in buffered substrate solutions was examined. The suspensions were tested for $\mathrm{H}_{2} \mathrm{~S}$ production from cysteine, cystine, homocystine, methionine, mercaptoacetate, sulphite, sulphate or thiosulphate. Some strains of the Proteus, Klebsiella, Salmonella, Arizona and Bethesda groups produced hydrogen sulphide when $10^{11}$ or more washed organisms $/ \mathrm{ml}$. were incubated at $37^{\circ}$ without an added source of sulphur. Generally tenfold greater quantities of bacteria were required to produce hydrogen sulphide within $24 \mathrm{hr}$. from homocystine, sulphite or thiosulphite as compared with cystine or cysteine. For the production of hydrogen sulphide from sodium mercaptoacetate, 10- and 100-fold larger quantities of bacteria were required. In the Brucella group all strains behaved alike, except two non-smooth strains which were spontaneously agglutinated in the buffer + substrate solutions and did not produce hydrogen sulphide. Among the Enterobacteriaceae the most active strains belonged to the Proteus group, the most inactive strains to the LargeSachs group. All strains of the Arizona and Bethesda groups, and some Klebsiella, Salmonella, Serratia and Providence strains multiplied on cysteine and cystine + buffer solutions, utilizing for growth the split products, pyruvic acid and ammonia.

Hydrogen sulphide production was inhibited by penicillin or streptomycin. Nonmultiplying micro-organisms exposed to penicillin, streptomycin, aureomycin or chloramphenicol for $24 \mathrm{hr}$. at $37^{\circ}$ remained viable, but lost their ability to produce hydrogen sulphide. This ability was regained on subculture. The hydrogen sulphide production of bacteria inactivated by penicillin was restored when the cells were removed by centrifugation, and residual penicillin destroyed by penicillinase. Addition of bacterial extracts heated at $50^{\circ}$ or pyridoxal phosphate, separately or together to inactivated bacteria, reactivated the hydrogen sulphide production of Proteus vulgaris. Bacterial extracts heated at $100^{\circ}$ exerted slight reactivating effects.

Hydrogen sulphide production by multiplying and non-multiplying bacteria has been described by many authors (see Clarke, 1953). Mention should be made of some investigations on factors which inhibit or stimulate the production of hydrogen sulphide. Beijerinck (1901) was the first to determine that many micro-organisms, among them escherichias and klebsiellas, were able to produce hydrogen sulphide from sulphite, thiosulphate or elementary sulphur, and that these reactions were more marked under anaerobic conditions. A further observation of Beijerinck (1904) was the 'indirect formation of sulphides' by Escherichia and Klebsiella spp., i.e. the biosynthesis of sulphurcontaining organic compounds from sulphate as $\mathbf{S}$ source and the production of sulphides by their decomposition. Braun \& Silberstein (1942) reported that hydrogen sulphide production from cystine, thiosulphate or elementary sulphur by Enterobacteriaceae was stimulated by the presence of glucose; the reaction 
was inhibited by pyruvate. Kallio \& Porter (1950) observed the anaerobic breakdown of cysteine to pyruvate, ammonia and hydrogen sulphide by Proteus vulgaris and described the reactivation of toluene-treated cells by the addition of boiled cell extract.

Kallio (1951) demonstrated the stimulation of the removal of $\mathrm{H}_{2} \mathrm{~S}$ from cysteine in Proteus sp. by pyridoxal phosphate, and Delwiche (1951) the stimulation of the same reaction in Escherichia coli by adenosine-5-phosphate, pyridoxal phosphate, biotin or $\alpha$-ketoglutarate, and its inhibition by phosphoglyceric acid, serine, alanine or semicarbazide. Ozek (1951) found that in the presence of different carbohydrates and alcohols different quantities of hydrogen sulphide were produced by the same strain. Skariton-Loewenthal, Rappaport \& Olitzki (1953) found that hydrogen sulphide production by Salmonella enteritidis on Kligler's iron agar (Difco) was inhibited by sodium biselenite and penicillin in concentrations which did not damage the vital functions of the bacterial cells.

\section{EXPERIMENTAL}

The organisms were grown for 2 days in Roux bottles on nutrient agar, then removed with saline, washed twice by centrifugation and resuspended in saline; $0.5 \mathrm{ml}$. of the required bacterial suspension was added to $0.5 \mathrm{ml}$. of the buffer + substrate solution $\left(0.15 \% \mathrm{~K}_{2} \mathrm{HPO}_{4} ; 0.05 \% \mathrm{KH}_{2} \mathrm{PO}_{4} ; 0.5 \% \mathrm{NaCl}\right.$; substrate). Inorganic substrates were added in a concentration of $0.5 \%$; organic substrates in a concentration of $0.1 \%$ or, if not soluble at this concentration, at saturation at $37^{\circ}$. The $\mathrm{pH}$ value of the mixtures was adjusted to 7.2. All experiments were carried out in $75 \times 11 \mathrm{~mm}$. test tubes firmly closed by rubber stoppers after insertion of lead acetate paper. The bacteria + substrate mixtures were incubated at $37^{\circ}$ and the beginning of $\mathrm{H}_{2} \mathrm{~S}$ production noted when blackening of the paper started. Observations were continued for $48 \mathrm{hr}$., although strongly positive reactions appeared in less than $1 \mathrm{hr}$.

The formation of soluble sulphides was tested by addition of $\mathrm{NaOH}$ and a reagent consisting of $0.1 \%$ lead acetate, $0.15 \%$ acetic acid and $0.25 \%$ gumarabic (Delwiche, 1951). This reagent occasionally gave weakly positive results when the reaction on lead acetate paper was completely negative. It did not give, however, satisfactory results in the presence of mercaptoacetate since by the mere addition of $\mathrm{NaOH}$, hydrogen sulphide was liberated from this substance. The results were usually first read after $30 \mathrm{~min}$. and then every $2 \mathrm{hr}$. until $6 \mathrm{hr}$. from the start of the experiment; 24 and $48 \mathrm{hr}$. readings were also taken. The velocity of the reaction was graded as follows: 3 , positive reaction after a few hours; 2 , positive at $24 \mathrm{hr}$; 1 , positive at $48 \mathrm{hr}$; 0 , negative reaction at $48 \mathrm{hr}$.

Another method used to measure relative activity of $\mathrm{H}_{2} \mathrm{~S}$ production was to dilute the original bacterial suspension containing $10^{11}$ organisms $/ \mathrm{ml}$. in twofold dilutions and to determine the minimal dilution able to produce a positive reaction within $24 \mathrm{hr}$. The lowest limit found was $10^{8}$ to $10^{9}$ micro-organisms/ ml. This method, however, revealed that certain Enterobacteriaceae were able to grow on cysteine or cystine + buffer solutions even with inocula of only 10 or 
100 organisms $/ \mathrm{ml}$. Growth and $\mathrm{H}_{2} \mathrm{~S}$ production were manifested by an increasing turbidity of the medium and a slowly increasing blackening of the lead acetate paper.

In all experiments a control tube was added which contained buffer solution without any sulphur-containing substrate and an inoculum of $10^{11}$ organisms/ $\mathrm{ml}$. This control was necessary since heavy suspensions of certain strains of the Proteus, Salmonella, Klebsiella and paracolon groups evolved hydrogen sulphide even in the absence of an added sulphur-containing compound, by the decomposition of their own sulphur-containing organic substances, as observed by Beijerinck (1904).

\section{RESULTS}

\section{Hydrogen sulphide production by Brucella spp.}

Table 1 shows that smooth strains of all three species of Brucella produced hydrogen sulphide from cysteine, cystine, homocystine, mercaptoacetate, $\mathrm{Na}_{2} \mathrm{SO}_{3}$ and $\mathrm{Na}_{2} \mathrm{~S}_{2} \mathrm{O}_{3}$. On homocystine, tenfold greater inocula were required; smooth strains of $B$. suis produced $\mathrm{H}_{2} \mathrm{~S}$ on this substrate more readily than the other species. On $\mathrm{Na}_{2} \mathrm{SO}_{3}$ and $\mathrm{Na}_{2} \mathrm{~S}_{2} \mathrm{O}_{3}$ the smooth strains of $B$. suis and $B$. abortus produced $\mathrm{H}_{2} \mathrm{~S}$ more quickly than the smooth strain of $B$. melitensis. On meth-

Table 1. $\mathrm{H}_{2} \mathrm{~S}$ production by washed brucellas on six different substrates

\begin{tabular}{|c|c|c|c|c|c|c|c|c|c|}
\hline & $\begin{array}{r}\text { Cystir } \\
\text { cyst }\end{array}$ & $\begin{array}{l}\text { ne and } \\
\text { eine }\end{array}$ & Homo & ystine & Merce & $\begin{array}{l}\text { apto- } \\
\text { ate }\end{array}$ & $\mathrm{Na}_{2} \mathrm{SO}$ & 3 and $\mathrm{N}$ & $\mathrm{a}_{2} \mathrm{~S}_{2} \mathrm{O}_{3}$ \\
\hline & & & & $\mathrm{Siz}$ & of inoc & ula & & & \\
\hline Strains & $3 \times 10^{10}$ & $\mathbf{3} \times 10^{9}$ & $3 \times 10^{11}$ & $3 \times 10^{10}$ & $3 \times 10^{10}$ & $3 \times 10^{9}$ & $3 \times 10^{10}$ & $3 \times 10^{9}$ & $3 \times 10^{8}$ \\
\hline & & & Rela & ive $\mathrm{H}_{2} \mathrm{~S}$ & product & ion obs & erved & & \\
\hline Smooth & & & & & & & & & \\
\hline B. melitensis, 5 & $\mathbf{3}$ & $1-2$ & 2 & 0 & 1 & $\mathbf{0}$ & 2 & 2 & $0-1$ \\
\hline B. melitensis, 7 & 3 & $0-1$ & $\mathbf{2}$ & 1 & 1 & $\mathbf{0}$ & 2 & 2 & $\mathbf{1}$ \\
\hline B. suis, 6 & $\mathbf{3}$ & 2 & 3 & $\mathbf{2}$ & 2 & 2 & 3 & 2 & $\mathbf{0}$ \\
\hline B. suis, 39 & $\mathbf{3}$ & 1-2 & $\mathbf{3}$ & $\mathbf{2}$ & 2 & $\mathbf{1}$ & $\mathbf{3}$ & $\mathbf{2}$ & $0-1$ \\
\hline B. abortus, 19 & 2-3 & $\mathbf{0}$ & 2 & $\mathbf{0}$ & 2 & 1 & $\mathbf{3}$ & 2 & $\mathbf{0}$ \\
\hline B. abortus, 2308 & $\mathbf{2}-\mathbf{3}$ & 0 & 2 & $0-1$ & 2 & 1 & 3 & $\mathbf{2}$ & $\mathbf{0}$ \\
\hline Non-smooth & & & & & & & & & \\
\hline B. melitensis, 7 & $\mathbf{0}$ & 0 & $\mathbf{0}$ & $\mathbf{0}$ & 2 & $\mathbf{0}$ & $\mathbf{2}$ & 1-2 & $\mathbf{0}$ \\
\hline B. suis, 6 & $\mathbf{2 - 3}$ & 1-2 & 2 & 2 & 2 & 1 & 3 & 2 & $0-1$ \\
\hline B. abortus, 2308 & $\mathbf{3}$ & 1 & 2 & 1 & 2 & 1 & $\mathbf{3}$ & 1-2 & $\mathbf{0}$ \\
\hline
\end{tabular}

\section{Smooth}

Streptomycin resistant

$\begin{array}{lcclllllll}\text { B. } \text { melitensis, 5 } & \mathbf{3} & \mathbf{2} & \mathbf{2} & \mathbf{2} & \mathbf{2} & \mathbf{1} & \mathbf{3} & \mathbf{2} & \mathbf{0} \\ \text { B. } \text { suis, 1* } & \mathbf{0} & \mathbf{0} & \mathbf{0} & \mathbf{0} & \mathbf{0} & \mathbf{0} & \mathbf{0} & \mathbf{0} & \mathbf{0} \\ \text { B. } \text { suis, 2* } & \mathbf{3} & \mathbf{2} & \mathbf{3} & \mathbf{0} & \mathbf{2} & \mathbf{1} & \mathbf{3} & \mathbf{2} & \mathbf{0} \\ \text { B. } \text { suis, 3* } & \mathbf{2} & \mathbf{0 - 1} & \mathbf{2} & \mathbf{0} & \mathbf{2} & \mathbf{1} & \mathbf{2} & \mathbf{1} & \mathbf{0} \\ \text { B. } \text { suis, 4** } & \mathbf{2} & \mathbf{0} & \mathbf{0} & \mathbf{0} & \mathbf{1} & \mathbf{1} & \mathbf{2} & \mathbf{0} & \mathbf{0} \\ \text { B. } \text { abortus, } 19 & \mathbf{3} & \mathbf{2} & \mathbf{2} & \mathbf{1} & \mathbf{2} & \mathbf{1} & \mathbf{3} & \mathbf{1} & \mathbf{0}\end{array}$

$3=$ strong $\mathrm{H}_{2} \mathrm{~S}$ production within $6 \mathrm{hr}$; $2=$ moderate $\mathrm{H}_{2} \mathrm{~S}$ production within $24 \mathrm{hr}$.; 1 = weak $\mathrm{H}_{2} \mathrm{~S}$ production $48 \mathrm{hr}$; $0=$ after $48 \mathrm{hr}$. incubation $\mathrm{H}_{2} \mathrm{~S}$ not produced. *=derived from $B$. suis 6 ; ** = derived from $B$. suis 39 . 
ionine only 4 strains, and on $\mathrm{Na}_{2} \mathrm{SO}_{4}$ only 3 strains out of 15 showed delayed weak reactions after $48 \mathrm{hr}$. when $3 \times 10^{11}$ organisms $/ \mathrm{ml}$. were used. The nonsmooth strains were strongly agglutinated in the buffer + substrate mixtures, particularly those which contained cysteine or homocystine. The non-smooth $B$. melitensis strain did not produce $\mathrm{H}_{2} \mathrm{~S}$ from these substrates. One of 4 streptomycin-resistant $B$. suis strains (non-smooth) lost completely its desulphhydrase activity for all substrates and another lost its activity for homocystine.

\section{Hydrogen-sulphide production by Enterobacteriaceae}

Tables 2 and 3 show that Proteus contains the most active $\mathrm{H}_{2} \mathrm{~S}$-producing species. All four species (31 strains) produced $\mathrm{H}_{2} \mathrm{~S}$ from almost all substrates examined, and only relatively small inocula were needed to yield a positive reaction within $24 \mathrm{hr}$. No Proteus strains tested were able to grow on the substrate + buffer mixture alone.

\section{Table 2. $\mathrm{H}_{2} \mathrm{~S}$ production by different species}

Inoculum : $10^{11}$ organisms $/ \mathrm{ml}$.

No. of strains producing $\mathrm{H}_{2} \mathrm{~S}$ within $24 \mathrm{hr}$. on

\begin{tabular}{|c|c|c|c|c|c|c|}
\hline Organism & $\begin{array}{c}\text { No. of } \\
\text { strains } \\
\text { examined }\end{array}$ & $\begin{array}{l}\text { Cysteine } \\
\text { and } \\
\text { cystine }\end{array}$ & $\begin{array}{l}\text { Homo- } \\
\text { cystine }\end{array}$ & $\begin{array}{l}\text { Mercapto- } \\
\text { acetate }\end{array}$ & $\mathrm{Na}_{8} \mathrm{SO}_{3}$ & $\mathrm{Na}_{2} \mathrm{~S}_{8} \mathrm{O}_{3}$ \\
\hline Proteus vulgaris & 15 & 15 & 15 & 7 & 15 & 15 \\
\hline P. mirabilis & 6 & 6 & 6 & 4 & 6 & 6 \\
\hline$P$. rettgeri & 5 & $\mathbf{5}$ & $\mathbf{2}$ & 1 & 5 & 1 \\
\hline P. morganii & $\mathbf{5}$ & $\mathbf{5}$ & 5 & $\mathbf{2}$ & 5 & $\mathbf{2}$ \\
\hline Providence group & 6 & 6 & 6 & 6 & 5 & 6 \\
\hline Klebsiella spp. & 12 & 12 & 8 & 9 & 12 & 12 \\
\hline Salmonella spp. & 12 & 12 & 4 & 6 & 9 & 10 \\
\hline Arizona group & 5 & $\mathbf{5}$ & 2 & 2 & $\mathbf{5}$ & $\mathbf{2}$ \\
\hline Bethesda-Ballerup & 6 & 6 & 2 & $\mathbf{2}$ & $\mathbf{3}$ & $\mathbf{2}$ \\
\hline Serratia spp. & $\mathbf{2}$ & 2 & 2 & $\mathbf{0}$ & 2 & $\mathbf{2}$ \\
\hline Escherichia spp. & 10 & 10 & $\mathbf{0}$ & $\mathbf{0}$ & 8 & 8 \\
\hline Alkalescens-dispar & 6 & 6 & 6 & $\mathbf{2}$ & 6 & 6 \\
\hline Shigella flexneri & 6 & 6 & $\mathbf{0}$ & $\mathbf{0}$ & $\mathbf{0}$ & $\mathbf{2}$ \\
\hline S. boydii & 8 & 8 & $\mathbf{3}$ & $\mathbf{0}$ & 2 & 3 \\
\hline S. sonnei & $\mathbf{3}$ & $\mathbf{3}$ & 2 & $\mathbf{0}$ & 2 & 2 \\
\hline $\begin{array}{l}\text { S. dysenteriae } \\
\text { type } 2 \text { (Schmitz) }\end{array}$ & 2 & 2 & $\mathbf{1}$ & $\mathbf{0}$ & $\mathbf{0}$ & 1 \\
\hline $\begin{array}{l}\text { S. dysenteriae } \\
\text { type } 1 \text { (Shiga) }\end{array}$ & 5 & $\mathbf{5}$ & $\mathbf{2}$ & $\mathbf{0}$ & $\mathbf{0}$ & $\mathbf{0}$ \\
\hline Large-Sachs group & 4 & 4 & $\mathbf{0}$ & $\mathbf{0}$ & $\mathbf{0}$ & $\mathbf{0}$ \\
\hline
\end{tabular}

Another group of organisms also proved to be very active, but these were able to grow on cysteine or cystine. All strains of the Arizona and BethesdaBallerup groups grew when inocula of $c .10 \mathrm{organisms} / \mathrm{ml}$. were used; other groups (Providence, Salmonella, Klebsiella, Serratia) contained some strains which were able to grow on cystine or cysteine. Among the salmonellas four organisms grew on cystine or cysteine as sole source of nitrogen, sulphur and energy, namely Salmonella paratyphi-B, $S$. cholerae-suis, $S$. typhi-murium and $S$. enteriditis. All these organisms were also able to grow on plain agar plates to which were added only pyruvate and ammonium sulphate. According to 
Kallio \& Porter, pyruvate, ammonia and $\mathrm{H}_{2} \mathrm{~S}$ are the enzymic split-products of cysteine. It seems, therefore, that these organisms were able to decompose cystine and cysteine and to use the products for growth. The Proteus strains did not grow in cysteine + buffer solution or in pyruvate + ammonium agar, but they grew when $1 \cdot 0 \mu \mathrm{g}$. nicotinamide $/ 100 \mathrm{ml}$. was also added.

Table 3. Minimal inocula required for hydrogen sulphide formation within 24 $h r$.

Organism

\section{Proteus vulgaris \\ $P$. mirabilis \\ P. rettgeri \\ P. morganii \\ Providence \\ Klebsiella spp. \\ Salmonella spp. \\ Arizona \\ Bethesda-Ballerup \\ Serratia spp.}

Escherichia spp.

Alkalescens-dispar

Shigella boydii

S. sonnei

S. flexneri

S. dysenteriae, type 2

S. dysenteriae, type 1

Large-Sachs

\section{Substrate}

$\overbrace{\begin{array}{c}\text { Cysteine } \\ \text { and } \\ \text { cystine }\end{array}}^{\begin{array}{l}\text { Homo- } \\ \text { cystine } \\ \text { Minimal inocula (cells/ml.) } \\ \text { acetate } \quad \mathrm{Na}_{2} \mathrm{SO}_{3}\end{array} \quad \mathrm{Na}_{2} \mathrm{~S}_{2} \mathrm{O}_{3}}$

\begin{tabular}{|c|c|c|c|c|}
\hline $2.5 \times 10^{8}$ & $2 \times 10^{9}$ & $10^{10}$ & $2 \times 10^{9}$ & $3 \times 10^{9}$ \\
\hline $1.5 \times 10^{8}$ & $5 \times 10^{8}$ & $10^{10}$ & $2 \times 10^{9}$ & $4 \times 10^{9}$ \\
\hline $2.5 \times 10^{8}$ & $4 \times 10^{2}$ & $10^{10}$ & $3 \times 10^{9}$ & $1 \times 10^{10}$ \\
\hline $3.5 \times 10^{8}$ & $2 \times 10^{9}$ & $10^{10}$ & $5 \times 10^{9}$ & $1 \times 10^{10}$ \\
\hline $\mathbf{G}$ or $2.5 \times 10^{8}$ & $4 \times 10^{9}$ & $10^{10}$ & $1 \times 10^{10}$ & $6 \times 10^{9}$ \\
\hline G or $2.5 \times 10^{8}$ & $1 \times 10^{10}$ & $10^{10}$ & $8 \times 10^{9}$ & $9 \times 10^{9}$ \\
\hline$G$ or $6.0 \times 10^{\theta}$ & $1 \times 10^{10}$ & $10^{11}$ & $4 \times 10^{10}$ & $2 \times 10^{10}$ \\
\hline $\mathbf{G}$ & $1 \times 10^{10}$ & $10^{10}$ & $1 \times 10^{10}$ & $1 \times 10^{10}$ \\
\hline $\mathbf{G}$ & $6 \times 10^{10}$ & $10^{10}$ & $1 \times 10^{10}$ & $1 \times 10^{10}$ \\
\hline G or $1.0 \times 10^{9}$ & $1 \times 10^{10}$ & $\mathbf{N}$ & $1 \times 10^{11}$ & $1 \times 10^{10}$ \\
\hline $7 \cdot 0 \times 10^{8}$ & $\mathbf{N}$ & $\mathbf{N}$ & $3 \times 10^{9}$ & $4 \times 10^{9}$ \\
\hline $1.0 \times 10^{9}$ & $1 \times 10^{10}$ & $10^{11}$ & $4 \times 10^{10}$ & $1 \times 10^{10}$ \\
\hline $4.5 \times 10^{10}$ & $1 \times 10^{11}$ & $\mathbf{N}$ & $1 \times 10^{11}$ & $1 \times 10^{11}$ \\
\hline $7 \cdot 5 \times 10^{9}$ & $1 \times 10^{11}$ & $\mathbf{N}$ & $1 \times 10^{11}$ & $5 \times 10^{10}$ \\
\hline $1.0 \times 10^{11}$ & $\mathbf{N}$ & $\mathbf{N}$ & $\mathbf{N}$ & $1 \times 10^{11}$ \\
\hline $1.0 \times 10^{9}$ & $1 \times 10^{11}$ & $\mathbf{N}$ & $\mathbf{N}$ & $1 \times 10^{11}$ \\
\hline $7 \cdot 0 \times 10^{9}$ & $1 \times 10^{11}$ & $\mathbf{N}$ & $\mathbf{N}$ & $\mathbf{N}$ \\
\hline $7 \cdot 5 \times 10^{10}$ & $\mathbf{N}$ & $\mathbf{N}$ & $\mathbf{N}$ & $\mathbf{N}$ \\
\hline
\end{tabular}

$\mathbf{G}=$ growth on the substrate + buffer solution;

$\mathrm{N}=\mathrm{H}_{2} \mathrm{~S}$ formation not observed after an incubation of $48 \mathrm{hr}$. with an inoculum of $10^{11}$ micro-organisms.

It should be mentioned that $\mathrm{H}_{2} \mathrm{~S}$ production by the bacteria on the substrate + buffer solution was not paralleled by their ability to produce $\mathrm{H}_{2} \mathrm{~S}$ when grown in Kligler's iron agar (Difco). Salmonella paratyphi-A showed almost the same activity on the buffer + substrate solution as did $S$. paratyphi-B, but on Kligler's iron agar only $S$. paratyphi-B yielded a positive $\mathrm{H}_{2} \mathrm{~S}$ reaction. A similar observation was made with three streptomycin-resistant strains of $S$. typh $i$ : on Kligler's iron agar they lost completely their ability to produce hydrogen sulphide (cf. Seligmann \& Wassermann, 1947) but in buffer + cystine, cysteine, mercaptoacetate, sulphite or thiosulphite they were almost as active as their streptomycin-sensitive parent strain.

With the Escherichia and alkalescens-dispar groups and the species Shigella flexneri, $S$. boydii, $S$. sonnei and $S$. dysenteriae type 2 , relatively large inocula were required to produce $\mathrm{H}_{2} \mathrm{~S}$ from cystine or cysteine; no strain grew on these compounds, few strains produced $\mathrm{H}_{2} \mathrm{~S}$ from homocystine (inoculum of $10^{11}$ organisms/ml.), and only two strains produced $\mathrm{H}_{2} \mathrm{~S}$ from mercaptoacetate. 
The least active organisms were Shigella dysenteriae type 1 (Shiga's bacillus) and the strains belonging to the Large-Sachs group. None produced $\mathrm{H}_{2} \mathrm{~S}$ from sulphite, thiosulphate or mercaptoacetate; two strains formed $\mathrm{H}_{2} \mathrm{~S}$ from homocystine. For $\mathrm{H}_{2} \mathrm{~S}$ production from cysteine or cystine $c .10^{11}$ organisms $/ \mathrm{ml}$. were required. None of the organisms belonging to the Shigella group produced $\mathrm{H}_{2} \mathrm{~S}$ on Kligler's iron agar.

\section{Penicillin inhibition of $\mathbf{H}_{2} \mathrm{~S}$ formation by non-multiplying cells in the presence of substrate}

Non-multiplying cells from three Brucella species were incubated, each with four different substrates and four different concentrations of penicillin; the results of this experiment are summarized in Table 4. In the presence of

Table 4. Inhibition by penicillin of $\mathrm{H}_{2} \mathrm{~S}$ production by brucellas

\begin{tabular}{|c|c|c|c|c|c|c|}
\hline \multirow[b]{2}{*}{ Strain } & \multirow[b]{2}{*}{ Substrates } & \multirow{2}{*}{$\begin{array}{c}\text { Penicillin } \\
\text { (u./ml.) }\end{array}$} & \multicolumn{4}{|c|}{ Reaction observed with inoculum of } \\
\hline & & & $1 \times 10^{10}$ & $5 \times 10^{9}$ & $2 \times 10^{9}$ & $1 \times 10^{8}$ \\
\hline \multirow[t]{8}{*}{ B. melitensis } & $\mathbf{C}$ & None & $\mathbf{3}$ & $\mathbf{2}$ & $\mathbf{2}$ & $0-1$ \\
\hline & $\mathbf{S}$ & & $\mathbf{3}$ & 2 & 2 & 2 \\
\hline & C & 200 & 3 & 2 & 0-1 & $0-1$ \\
\hline & $\mathbf{S}$ & & $\mathbf{3}$ & 2 & $\mathbf{2}$ & 1 \\
\hline & $\mathbf{C}$ & 2,000 & 2 & $0-1$ & $\mathbf{0}$ & $\mathbf{0}$ \\
\hline & $\mathbf{S}$ & & 2 & $0-1$ & $\mathbf{0}$ & $\mathbf{0}$ \\
\hline & $\mathbf{C}$ & 20,000 & $\mathbf{0}$ & $\mathbf{0}$ & $\mathbf{0}$ & $\mathbf{0}$ \\
\hline & $\mathbf{S}$ & & $0-1$ & 0 & $\mathbf{0}$ & 0 \\
\hline \multirow[t]{8}{*}{ B. suis } & $\mathbf{C}$ & None & $\mathbf{3}$ & $2-3$ & $2-3$ & 2 \\
\hline & $\mathbf{S}$ & & $\mathbf{3}$ & $\mathbf{2}$ & 2 & $1-2$ \\
\hline & C & 200 & $\mathbf{3}$ & 2-3 & 2 & 2 \\
\hline & $\mathbf{S}$ & & 3 & 2 & 2 & 2 \\
\hline & $\mathbf{C}$ & 2,000 & $\mathbf{3}$ & 1-2 & $0-1$ & $\mathbf{0}$ \\
\hline & $\mathbf{S}$ & & 2 & 2 & 2 & $0-1$ \\
\hline & C & 20,000 & $\mathbf{0}$ & $\mathbf{0}$ & $\mathbf{0}$ & $\mathbf{0}$ \\
\hline & $\mathbf{S}$ & & $0-1$ & $\mathbf{0}$ & $\mathbf{0}$ & $\mathbf{0}$ \\
\hline \multirow[t]{8}{*}{ B. abortus } & C & None & 2-3 & 1-2 & 1 & 0 \\
\hline & $\mathbf{S}$ & & 8 & $\mathbf{2}$ & 2 & $1-2$ \\
\hline & C & 200 & 2 & 1-2 & 1-2 & 0 \\
\hline & $\mathbf{S}$ & & $\mathbf{3}$ & 2 & $\mathbf{2}$ & 1-2 \\
\hline & $\mathbf{C}$ & 2,000 & 1-2 & $\mathbf{0}$ & $\mathbf{0}$ & 0 \\
\hline & $\mathbf{S}$ & & $\mathbf{3}$ & 2 & 1-2 & 0 \\
\hline & $\mathbf{C}$ & 20,000 & $\mathbf{0}$ & $\mathbf{0}$ & 0 & 0 \\
\hline & $\mathbf{S}$ & & o & 0 & $\mathbf{0}$ & 0 \\
\hline
\end{tabular}

$\mathrm{C}=$ cystine or cysteine; $\mathrm{S}=\mathrm{Na}_{2} \mathrm{SO}_{3}$ or $\mathrm{Na}_{2} \mathrm{~S}_{2} \mathrm{O}_{3} .3,2,1,0$ for relative $\mathrm{H}_{2} \mathrm{~S}$ production have the same significance as in Table 1.

20,000 units penicillin $/ \mathrm{ml} . \mathrm{H}_{2} \mathrm{~S}$ production from cysteine, cystine, sulphite or thiosulphate was completely inhibited. In the presence of 2000 units penicillin/ml. a marked decrease of $\mathrm{H}_{2} \mathrm{~S}$ production was observed when smaller inocula were used. This inhibition by penicillin was more marked with cysteine or cystine than with thiosulphate or sulphite.

After incubating two suspensions $\left(10^{10}\right.$ and $5 \times 10^{9}$ organisms $/ \mathrm{ml}$.) with 
20,000 units penicillin $/ \mathrm{ml}$. and the above-mentioned substrates for $48 \mathrm{hr}$. the cells were removed by centrifugation, washed in buffer solution and resuspended in the original substrate + buffer solutions without penicillin. All three organisms (Brucella melitensis, B. suis, B. abortus) with both sizes of inoculum showed $\mathrm{H}_{2} \mathrm{~S}$ production from cysteine or cystine but not from sulphite or thiosulphate. It seemed, therefore, that some enzyme or enzymes which acted on the latter substrates had been destroyed by penicillin, while the enzymes concerned with cysteine and cystine were only inhibited by penicillin and restored to activity when it was removed. The viability of the cells was not damaged by the exposure to penicillin.

\section{Effect of streptomycin on $\mathrm{H}_{2} \mathrm{~S}$ production}

One strain, Brucella abortus 2308, was found in which the cysteine desulphhydrase appeared to be more sensitive to streptomycin than the enzymes which produced $\mathrm{H}_{2} \mathrm{~S}$ from the other substrates tested. The cells were exposed to different concentrations of streptomycin and the required substrate. $\mathrm{H}_{2} \mathrm{~S}$ production from sulphite or thiosulphate remained active even in the presence of $100,000 \mu \mathrm{g}$. streptomycin $/ \mathrm{ml}$., but $\mathrm{H}_{2} \mathrm{~S}$ production from cystine or cysteine ceased in the presence of $1000 \mu \mathrm{g} . / \mathrm{ml}$. (Table 5). The cells were allowed to

Table 5. Inhibition of $\mathrm{H}_{2} \mathrm{~S}$ production by Brucella abortus 2308 by streptomycin

\begin{tabular}{|c|c|c|c|}
\hline \multicolumn{4}{|c|}{$\begin{array}{l}\text { Substrates: cysteine and cystine. } \\
\text { Inoculum size (cells/ml.) }\end{array}$} \\
\hline & $10^{10}$ & $5 \times 10^{9}$ & $2 \times 10^{9}$ \\
\hline $\begin{array}{l}\text { Streptomycin } \\
(\mu \mathrm{g} . / \mathrm{ml} .)\end{array}$ & \multicolumn{3}{|c|}{ Relative $\mathbf{H}_{2} \mathbf{S}$ production } \\
\hline None & $\mathbf{3}$ & $\mathbf{3}$ & 2 \\
\hline 10 & 3 & 3 & 2 \\
\hline 100 & 2 & 2 & 2 \\
\hline 1,000 & 1 & 0 & 0 \\
\hline 10,000 & 0 & 0 & 0 \\
\hline 100,000 & $\mathbf{0}$ & $\mathbf{0}$ & 0 \\
\hline
\end{tabular}

remain in contact with substrate $+100,000 \mu \mathrm{g}$. streptomycin $/ \mathrm{ml}$. for $48 \mathrm{hr}$, then removed by centrifugation, washed in buffer solution and resuspended in the substrate-buffer solution; $\mathrm{H}_{2} \mathrm{~S}$ was then no longer produced. A similar experiment was carried out with Salmonella typhi 0901 and three streptomycin-resistant mutants derived from it. The results, summarized in Table 6, showed that washed suspensions of strain 0901 and its three streptomycinresistant mutants produced $\mathrm{H}_{2} \mathrm{~S}$ from cysteine; on Kligler's iron agar, only the original strain produced $\mathrm{H}_{2} \mathrm{~S}$, while the streptomycin-dependent mutants failed to do so. The desulphhydrase activity of all four strains was markedly inhibited by $20,000 \mu \mathrm{g}$. streptomycin $/ \mathrm{ml}$. When the cells were removed by centrifugation, washed and again resuspended in the cysteine + buffer solution, $\mathrm{H}_{2} \mathrm{~S}$ production was not restored, while in a control abundant $\mathrm{H}_{2} \mathrm{~S}$ production occurred. 
Table 6. Inhibition by streptomycin of $\mathrm{H}_{2} \mathrm{~S}$ production by Salmonella typhi 0901 and three streptomycin-resistant mutants

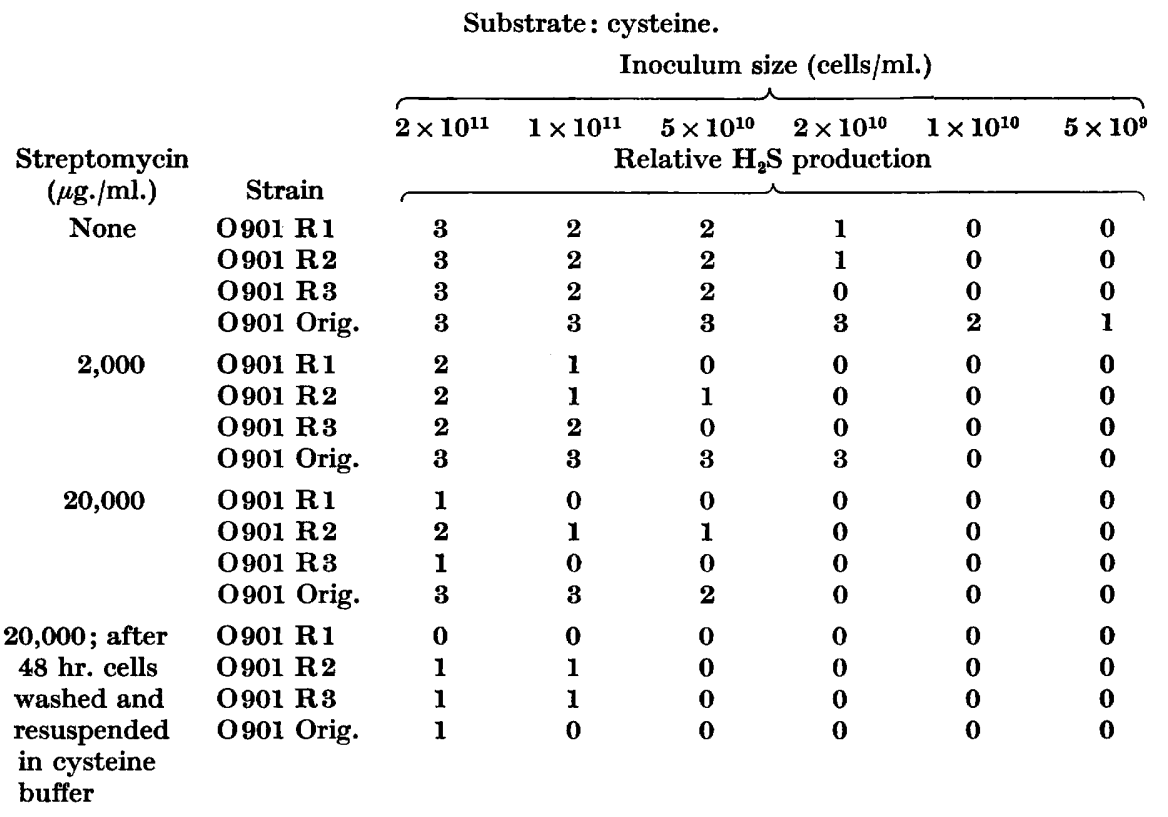

Inactivation of the hydrogen sulphide-producing enzymes by exposure

In the earlier experiments bacteria had been brought in contact with sulphurcontaining substrates and antibiotics simultaneously. It was therefore not clear whether the antibiotics inactivated the $\mathrm{H}_{2} \mathrm{~S}$-producing enzymes or whether the sulphur-containing substrates prevented the action of the antibiotics. To exclude any mutual influence the technique in the following experiments was changed. The bacteria were exposed for $24 \mathrm{hr}$. at $37^{\circ}$ to different concentrations of antibiotics, washed twice in saline and then resuspended in the buffer + substrate solution. The experiments presented in Table 7 show that an exposure to 20,000 units penicillin $/ \mathrm{ml}$. inactivated the $\mathrm{H}_{2} \mathrm{~S}$-producing enzymes of all strains under experiment. Aureomycin $(1.0 \mathrm{mg} . / \mathrm{ml}$.) acted in the same way, but did not prevent hydrogen sulphide production from thiosulphate by Brucella suis. Chloramphenicol $(1.0 \mathrm{mg} . / \mathrm{ml}$.) or streptomycin $\left(0 \cdot 1 \mathrm{mg}\right.$./ml.) prevented $\mathrm{H}_{2} \mathrm{~S}$-production by $B$. melitensis and two Salmonella typhi strains, while Brucella abortus and $B$. suis remained active.

\section{Reactivation of $\mathrm{H}_{2} \mathrm{~S}$-producing enzymes by subcultivation}

The experiment presented in Table 8 was designed to show whether this inactivation was permanent or whether the $\mathrm{H}_{2} \mathrm{~S}$-producing enzyme could be reactivated by subcultivation of the inactivated bacteria. The bacteria (two Salmonella typhi strains) were exposed to inactivating concentrations of antibiotics and then subcultured on plain agar, on casein hydrolysed by acid with or without $0.01 \%$ cysteine, or with $0.05 \%$ sodium thiosulphate. After one or 
two subcultures the $\mathrm{H}_{2} \mathrm{~S}$-producing activity was again restored. Before the subcultures were started, all bacterial suspensions were counted by plating. Suspensions of $S$. typhi strains 0901 and Ty 2 contained almost the same number of viable organisms whether or not they had been kept in buffer

Table 7. Inactivation of hydrogen sulphide producing enzymes by exposure of bacteria to antibiotics

\begin{tabular}{|c|c|c|c|c|c|c|c|c|c|c|c|}
\hline & \multirow[b]{3}{*}{$\begin{array}{c}\text { Concentra- } \\
\text { tion } \\
(\mathrm{mg} . / \mathrm{ml} .)\end{array}$} & \multicolumn{10}{|c|}{ Substrate } \\
\hline & & \multicolumn{4}{|c|}{ Cysteine } & \multicolumn{2}{|c|}{ Strain } & \multicolumn{3}{|c|}{$\mathrm{Na}_{2} \mathrm{~S}_{2} \mathrm{O}_{3}$} & \multirow[b]{2}{*}{$\mathbf{E}$} \\
\hline Antibiotic & & $\mathbf{A}$ & $\mathbf{B}$ & $\mathbf{C}$ & $\underset{\text { Relativ }}{\text { D }}$ & $\begin{array}{l}\mathrm{E} \\
\text { ve } \mathrm{H}_{\mathbf{2}} \mathrm{S}\end{array}$ & $\underset{\text { produ }}{\text { A }}$ & $\underset{\text { ction }}{\mathbf{B}}$ & $\mathbf{C}$ & $\mathbf{D}$ & \\
\hline Aureomycin & $\begin{array}{l}1 \cdot 0 \\
0 \cdot 1\end{array}$ & $\begin{array}{l}\mathbf{0} \\
\mathbf{0}\end{array}$ & $\begin{array}{l}\mathbf{0} \\
\mathbf{3}\end{array}$ & $\begin{array}{l}\mathbf{0} \\
\mathbf{3}\end{array}$ & $\begin{array}{l}0 \\
0\end{array}$ & $\begin{array}{l}\mathbf{0} \\
\mathbf{3}\end{array}$ & $\begin{array}{l}\mathbf{0} \\
\mathbf{0}\end{array}$ & $\begin{array}{l}\mathbf{3} \\
\mathbf{3}\end{array}$ & $\begin{array}{l}\mathbf{0} \\
\mathbf{3}\end{array}$ & $\begin{array}{l}\mathbf{0} \\
\mathbf{0}\end{array}$ & $\begin{array}{l}\mathbf{0} \\
\mathbf{2}\end{array}$ \\
\hline Chloramphenicol & $\begin{array}{l}1 \cdot 0 \\
0 \cdot 1\end{array}$ & $\begin{array}{l}\mathbf{0} \\
\mathbf{0}\end{array}$ & $\begin{array}{l}\mathbf{3} \\
\mathbf{3}\end{array}$ & $\begin{array}{l}\mathbf{3} \\
\mathbf{3}\end{array}$ & $\begin{array}{l}0 \\
0\end{array}$ & $\begin{array}{l}\mathbf{0} \\
\mathbf{2}\end{array}$ & $\begin{array}{l}\mathbf{0} \\
\mathbf{0}\end{array}$ & $\begin{array}{l}\mathbf{3} \\
\mathbf{8}\end{array}$ & $\begin{array}{l}\mathbf{3} \\
\mathbf{3}\end{array}$ & $\begin{array}{l}\mathbf{0} \\
0\end{array}$ & $\begin{array}{l}\mathbf{0} \\
\mathbf{0}\end{array}$ \\
\hline Streptomycin & $\begin{array}{l}0.1 \\
0.01 \\
0.001\end{array}$ & $\begin{array}{l}\mathbf{0} \\
\mathbf{3} \\
\mathbf{3}\end{array}$ & $\begin{array}{l}\mathbf{3} \\
\mathbf{3} \\
\mathbf{3}\end{array}$ & $\begin{array}{l}\mathbf{3} \\
\mathbf{3} \\
\mathbf{3}\end{array}$ & $\begin{array}{l}\mathbf{0} \\
\mathbf{3} \\
\mathbf{3}\end{array}$ & $\begin{array}{l}\mathbf{0} \\
\mathbf{3} \\
\mathbf{3}\end{array}$ & $\begin{array}{l}\mathbf{0} \\
\mathbf{3} \\
\mathbf{3}\end{array}$ & $\begin{array}{l}\mathbf{3} \\
\mathbf{3} \\
\mathbf{3}\end{array}$ & $\begin{array}{l}\mathbf{3} \\
\mathbf{3} \\
\mathbf{3}\end{array}$ & $\begin{array}{l}\mathbf{0} \\
\mathbf{0} \\
\mathbf{3}\end{array}$ & $\begin{array}{l}\mathbf{0} \\
\mathbf{2} \\
\mathbf{3}\end{array}$ \\
\hline Penicillin & $\begin{array}{r}\text { (u. } / \mathrm{ml}) \text { ) } \\
20,000 \\
2,000 \\
200\end{array}$ & $\begin{array}{l}\mathbf{0} \\
\mathbf{2} \\
\mathbf{3}\end{array}$ & $\begin{array}{l}\mathbf{0} \\
\mathbf{0} \\
\mathbf{3}\end{array}$ & $\begin{array}{l}\mathbf{0} \\
\mathbf{0} \\
\mathbf{2}\end{array}$ & $\begin{array}{l}\mathbf{0} \\
\mathbf{3} \\
\mathbf{3}\end{array}$ & $\begin{array}{l}\mathbf{0} \\
\mathbf{3} \\
\mathbf{3}\end{array}$ & $\begin{array}{l}\mathbf{0} \\
\mathbf{0} \\
\mathbf{3}\end{array}$ & $\begin{array}{l}\mathbf{0} \\
\mathbf{3} \\
\mathbf{8}\end{array}$ & $\begin{array}{l}\mathbf{0} \\
\mathbf{3} \\
\mathbf{3}\end{array}$ & $\begin{array}{l}\mathbf{0} \\
\mathbf{0} \\
\mathbf{3}\end{array}$ & $\begin{array}{l}\mathbf{0} \\
\mathbf{0} \\
\mathbf{3}\end{array}$ \\
\hline
\end{tabular}

Bacteria $=10^{11} \mathrm{ml} . \quad \mathrm{A}=\mathrm{B}$. melitensis; $\mathrm{B}=\mathrm{B}$. suis; $\mathrm{C}=\mathrm{B}$. abortus; $\mathrm{D}=\mathrm{S}$. typhi (0901); $\mathbf{E}=S$. typhi (Ty2).

solution or exposed to antibiotics; the respective counts for the two strains were $6.5 \times 10^{11}$ and $8.0 \times 10^{11}$ organisms $/ \mathrm{ml}$. This experiment proved, therefore, that although the antibiotics did not decrease the viability of resting cells, they damaged their $\mathrm{H}_{2} \mathrm{~S}$-producing enzymes.

\section{Inactivation of the anti-desulphhydrase activity of penicillin by penicillinase}

Penicillin (25,000 units/ml.) was left in contact for different periods with different concentrations of penicillinase (Nutritional Biochemicals Corporation, Cleveland, Ohio). Bacterial suspensions of $10^{11}$ organisms $/ \mathrm{ml}$. were exposed for $24 \mathrm{hr}$. to the products of this reaction. The organisms were removed from the penicillin + penicillinase mixture by centrifugation, washed in buffer solution and resuspended in cysteine + buffer solution. The results obtained with Proteus vulgaris and Brucella abortus (strain 19) are summarized in Table 9 which shows that a contact of 25,000 units penicillin $/ \mathrm{ml}$. with $0.2 \%$ pencillinase for $2 \mathrm{hr}$. was long enough to inactivate the anti-desulphhydrase activity against Proteus vulgaris, while for Brucella abortus even $\mathbf{0} \cdot 1 \%$ penicillinase was sufficient. No further changes occurred when the activity of the penicillin and penicillinase mixtures was tested after $4 \mathrm{hr}$. contact. 
Table 8. Reactivation of hydrogen sulphide production by subculture on antibiotic-free medium

Effect of $24 \mathrm{hr}$. exposure, $\mathrm{H}_{2} \mathrm{~S}$ production on

No. of subcultures required for reactivation on medium

\begin{tabular}{|c|c|c|c|c|c|c|c|c|c|c|c|}
\hline \multirow{2}{*}{$\begin{array}{l}\text { Strain } \\
\text { tested }\end{array}$} & \multirow{2}{*}{$\begin{array}{c}\text { Antibiotic } \\
\text { (concentration/ } \\
\text { ml.) }\end{array}$} & \multirow{2}{*}{$\begin{array}{l}\text { Cyst- } \\
\text { eine } \\
\text { (C) }\end{array}$} & \multirow{2}{*}{$\begin{array}{c}\mathrm{Na}_{2} \mathrm{~S}_{2} \mathrm{O}_{3} \\
(\mathrm{~T})\end{array}$} & \multicolumn{2}{|c|}{$\mathbf{I}$} & \multicolumn{2}{|c|}{ II } & \multicolumn{2}{|c|}{ III } & \multicolumn{2}{|c|}{ IV } \\
\hline & & & & C & $\mathbf{T}$ & $\mathbf{C}$ & $\mathbf{T}$ & C & $\mathbf{T}$ & C & $\mathbf{T}$ \\
\hline \multirow{8}{*}{$\begin{array}{l}\text { S. typhi } \\
0901\end{array}$} & Penicillin & & & & & & & & & & \\
\hline & $\begin{array}{c}20,000 \mathrm{u} . \\
\text { Streptomycin }\end{array}$ & 0* & 0* & 1 & 1 & 1 & 2 & $\mathbf{1}$ & $\mathbf{1}$ & 1 & $\mathbf{1}$ \\
\hline & $0.2 \mathrm{mg}$. & $\mathbf{0}$ & 0 & 1 & 2 & 2 & $\mathbf{2}$ & 2 & $\mathbf{2}$ & $\mathbf{2}$ & 1 \\
\hline & $\begin{array}{c}0.02 \mathrm{mg} . \\
\text { Aureomycin }\end{array}$ & 2 & $\mathbf{0}$ & - & 2 & . & 1 & . & 1 & - & 1 \\
\hline & $1.0 \mathrm{mg}$. & $\mathbf{0}$ & $\mathbf{0}$ & 1 & 2 & 2 & 2 & 2 & 2 & 2 & 2 \\
\hline & $0.1 \mathrm{mg}$. & $\mathbf{0}$ & o & 1 & $\mathbf{2}$ & 1 & 2 & 1 & $\mathbf{2}$ & 2 & $\mathbf{2}$ \\
\hline & $\begin{array}{l}1.0 \mathrm{mg} \text {. } \\
\text { Control }\end{array}$ & $\mathbf{0}$ & 0 & 1 & 2 & 1 & 2 & 1 & 1 & $\mathbf{1}$ & 1 \\
\hline & (Buffer) & 3 & 3 & - & . & . & . & - & - & - & - \\
\hline \multirow[t]{6}{*}{$\begin{array}{l}\text { S. typhi } \\
\text { Ty2 }\end{array}$} & $\begin{array}{l}\text { Penicillin } \\
\text { 20,000 u. } \\
\text { Streptomycin }\end{array}$ & $\mathbf{0}$ & 0 & 1 & 1 & 1 & 2 & 1 & $\mathbf{1}$ & 1 & $\mathbf{1}$ \\
\hline & $\begin{array}{c}0.2 \mathrm{mg} \text {. } \\
\text { Aureomycin }\end{array}$ & 0 & 0 & 1 & 2 & 2 & 2 & 2 & 2 & 2 & 2 \\
\hline & $1.0 \mathrm{mg}$. & 0 & 0 & 1 & 2 & 2 & 2 & 2 & 2 & 2 & 2 \\
\hline & $\begin{array}{l}0.1 \mathrm{mg} \text {. } \\
\text { Chloromycetin }\end{array}$ & $\mathbf{0}$ & $\mathbf{0}$ & 1 & 2 & 1 & 2 & 1 & $\mathbf{2}$ & $\mathbf{1}$ & 1 \\
\hline & $\begin{array}{l}1.0 \mathrm{mg} . \\
\text { Control }\end{array}$ & $\mathbf{0}$ & 0 & 1 & $\mathbf{2}$ & 1 & 2 & $\mathbf{1}$ & 1 & 1 & 1 \\
\hline & (Buffer) & 3 & 3 & - & - & - & - & - & - & - & - \\
\hline
\end{tabular}

Medium $I=$ plain agar; medium $I I=$ acid hydrolysed casein; medium $I I I=a s$ II with cysteine $0.01 \%$; medium IV =as II with $\mathrm{Na}_{2} \mathrm{~S}_{2} \mathrm{O}_{3} 0.05 \%$.

* Figures in these columns have the same significance as in Table 1.

$\mathbf{C}=$ cysteine as substrate; $\mathbf{T}=$ thiosulphate as substrate.

Table 9. Inactivation of anti-desulphhydrase activity of penicillin by penicillinase

Test performed with $B$. abortus and $P$. vulgaris on cysteine $\left(10^{11}\right.$ cells $/ \mathrm{ml}$.).

Concentration of penicillinase

\begin{tabular}{|c|c|c|c|c|c|c|}
\hline $\begin{array}{l}\text { Time of } \\
\text { reaction }\end{array}$ & Test & \multicolumn{5}{|c|}{$\begin{array}{l}\text { Relative } \mathrm{H}_{2} \mathrm{~S} \text { production after contact with the } \\
\text { product of the penicillin-penicillinase reaction }\end{array}$} \\
\hline 2 & $\begin{array}{l}P . \text { vulgaris } \\
\text { B. abortus }\end{array}$ & $\begin{array}{l}\mathbf{2} \\
\mathbf{2}\end{array}$ & $\begin{array}{l}\mathbf{2} \\
\mathbf{2}\end{array}$ & $\begin{array}{l}\mathbf{0} \\
\mathbf{2}\end{array}$ & $\begin{array}{l}0 \\
0\end{array}$ & $\begin{array}{l}\mathbf{0} \\
\mathbf{0}\end{array}$ \\
\hline 4 & $\begin{array}{l}\text { P. vulgaris } \\
\text { B. abortus }\end{array}$ & $\begin{array}{l}\mathbf{2} \\
\mathbf{2}\end{array}$ & $\begin{array}{l}\mathbf{2} \\
\mathbf{2}\end{array}$ & $\begin{array}{l}\mathbf{0} \\
\mathbf{2}\end{array}$ & $\begin{array}{l}0 \\
0\end{array}$ & $\begin{array}{l}\mathbf{0} \\
\mathbf{0}\end{array}$ \\
\hline
\end{tabular}

The minimal time of contact with penicillin required for the inactivation of bacterial desulphhydrases

Organisms $\left(10^{11} / \mathrm{ml}\right.$.) were exposed for different times to 25,000 units penicillin $/ \mathrm{ml}$. at $37^{\circ}$, centrifuged, washed in buffer solution, resuspended in substrate + buffer solution and examined for $\mathrm{H}_{2} \mathrm{~S}$ production. The results, 
summarized in Table 10, show that time intervals from 5 to $20 \mathrm{hr}$. had to elapse before complete inactivation was reached. After an exposure to penicillin for $20 \mathrm{hr}$. all five organisms were completely inactivated.

Table 10. Inactivation of $\mathrm{H}_{2} \mathrm{~S}$ formation by bacterial suspensions exposed to penicillin Suspensions : $10^{11}$ cells/ml. Penicillin 25,000 u./ml. Temp. 37 ${ }^{\circ}$.

\begin{tabular}{|c|c|c|c|c|c|c|}
\hline \multirow[b]{2}{*}{$\begin{array}{l}\text { Substrate } \\
\text { tested }\end{array}$} & \multirow{2}{*}{$\begin{array}{c}\text { Exposure } \\
\text { (hr.) }\end{array}$} & \multicolumn{5}{|c|}{ Relative $\mathrm{H}_{2} \mathrm{~S}$ production observed with strain } \\
\hline & & $\mathbf{A}$ & $\mathbf{B}$ & $\mathbf{C}$ & $\mathbf{D}$ & $\mathbf{E}$ \\
\hline \multirow[t]{6}{*}{ Cysteine } & $\mathbf{2 \cdot 5}$ & 2 & $\mathbf{3}$ & $\mathbf{3}$ & $\mathbf{3}$ & $\mathbf{3}$ \\
\hline & $5 \cdot 0$ & 1 & 3 & $\mathbf{3}$ & $\mathbf{3}$ & $\mathbf{1}$ \\
\hline & $10 \cdot 0$ & 0 & $\mathbf{3}$ & $\mathbf{3}$ & $\mathbf{3}$ & 0 \\
\hline & $15 \cdot 0$ & $\mathbf{0}$ & $\mathbf{2}$ & 2 & $\mathbf{0}$ & $\mathbf{0}$ \\
\hline & $20 \cdot 0$ & $\mathbf{0}$ & 0 & $\mathbf{0}$ & 0 & $\mathbf{0}$ \\
\hline & $\begin{array}{l}\text { Control not } \\
\text { exposed }\end{array}$ & $\mathbf{3}$ & $\mathbf{3}$ & 3 & $\mathbf{3}$ & 3 \\
\hline \multirow[t]{6}{*}{ Sodium thiosulphate } & $\mathbf{2 \cdot 5}$ & $\mathbf{0}$ & 2 & 2 & $\mathbf{3}$ & $\mathbf{3}$ \\
\hline & $\mathbf{5 \cdot 0}$ & $\mathbf{0}$ & $\mathbf{2}$ & $\mathbf{0}$ & $\mathbf{3}$ & $\mathbf{3}$ \\
\hline & $10 \cdot 0$ & $\mathbf{0}$ & $\mathbf{0}$ & $\mathbf{0}$ & $\mathbf{3}$ & $\mathbf{3}$ \\
\hline & $15 \cdot 0$ & $\mathbf{0}$ & 0 & $\mathbf{0}$ & 0 & 0 \\
\hline & $20 \cdot 0$ & $\mathbf{0}$ & $\mathbf{0}$ & $\mathbf{0}$ & $\mathbf{0}$ & $\mathbf{0}$ \\
\hline & $\begin{array}{l}\text { Control not } \\
\text { exposed }\end{array}$ & 1 & $\mathbf{3}$ & 2 & $\mathbf{3}$ & $\mathbf{3}$ \\
\hline
\end{tabular}

$\mathbf{A}=$ S. typhi $; \mathbf{B}=\boldsymbol{P}$. vulgaris $; \mathrm{C}=\boldsymbol{P}$. morganii $; \mathrm{D}=\boldsymbol{B}$. abortus (strain 19 ) $; \mathbf{E}=\mathbf{B}$. abortus (strain 2308).

\section{Reactivation of the desulphhydrase activity by penicillinase}

The experiments described above showed that under the conditions used, the anti-desulphhydrase action of penicillin was destroyed by penicillinase within 2-4 hr. An attempt was made to determine the minimal time of contact between penicillin and bacteria required to inactivate the desulphhydrase, by addition of penicillinase at different times after mixing penicillin and bacteria. The addition of the penicillinase effected a reactivation of the bacterial desulphhydrases while washing left the bacteria inactive.

Table 11 shows that the bacterial desulphhydrase activity was not reactivated by addition of the substrate to the penicillin + bacteria mixture or by washing and resuspension in the substrate + buffer solution. However, when substrate + penicillinase were added, some traces of hydrogen sulphide

Table 11. Penicillinase reactivation of penicillin-inactivated suspensions Suspension: $10^{11}$ bacteria/ml. exposed $20 \mathrm{hr}$. to $25,000 \mathrm{u}$. penicillin $/ \mathrm{ml}$.

Treatment of the bacteria after $24 \mathrm{hr}$. exposure to penicillin

(1) Cysteine and penicillinase (0.5\%) added

(2) Cysteine added

(3) Bacteria washed, then cysteine and penicillinase $(0.5 \%)$ added

(4). Bacteria washed, then cysteine added
Relative $\mathrm{H}_{2} \mathrm{~S}$ production of

$\begin{array}{ccc}\text { S. typhi } & \text { P. vulgaris } & \text { B. abortus } \\ 1 & 1 & 1 \\ 0 & 0 & 0 \\ 2 & 2 & 2 \\ 0 & 0 & 0\end{array}$


appeared after $48 \mathrm{hr}$. When the bacteria were washed and the substrate and penicillinase added, the reactivation was complete and within $24 \mathrm{hr}$. hydrogen sulphide was produced. It seems, therefore, that centrifugation and washing alone did not remove all traces of penicillin. On the other hand, washing followed by penicillinase treatment reactivated the bacterial cells, proving that the changes exerted by penicillin were reversible. Washing alone, even repeated five times, did not exert this effect.

Since the penicillinase solutions were partly inactivated by filtration through Seitz filters, we compared in the following experiments the reactivating action of different concentrations of both filtered and non-filtered penicillinase solutions. Table 12 shows that with 0.2 and $0.1 \%$ non-filtered, and with 0.5 and $0 \cdot 2 \%$ filtered solutions of penicillinase no reactivation of cysteine desulphhydrase was obtained unless the bacteria were washed and then brought in contact with penicillinase + cysteine. Similar results were obtained with sodium thiosulphate instead of cysteine. Higher concentrations of nonfiltered penicillinase solutions were not employed, since higher concentrations of this enzyme preparation alone when brought in contact with cysteine themselves produced hydrogen sulphide after $48 \mathrm{hr}$. In order to exclude this source of error the maximal concentration used was $0 \cdot 2 \%$.

Table 12. Reactivating effect of different types of penicillinase solutions Suspensions $10^{11}$ bacteria/ml. inactivated by $25,000 \mathrm{u}$. penicillin $/ \mathrm{ml}$. Penicillinase

\begin{tabular}{|c|c|c|c|c|c|c|c|}
\hline \multirow{3}{*}{$\begin{array}{c}\text { Treatment after } \\
\text { inactivation }\end{array}$} & & & & & & & \\
\hline & \multirow[b]{2}{*}{ Type } & \multirow{2}{*}{$\begin{array}{c}\text { Concen- } \\
\text { tration } \\
(\%)\end{array}$} & \multicolumn{5}{|c|}{ Relative $\mathrm{H}_{2} \mathrm{~S}$ production by strain } \\
\hline & & & $\mathbf{A}$ & $\mathbf{B}$ & $\mathbf{C}$ & $\mathbf{D}$ & $\mathbf{E}$ \\
\hline \multirow[t]{4}{*}{ Penicillinase added } & Not filtered & $0 \cdot 2$ & $\mathbf{0}$ & $\mathbf{0}$ & 1 & $\mathbf{0}$ & $\mathbf{0}$ \\
\hline & & $0 \cdot 1$ & $\mathbf{0}$ & $\mathbf{0}$ & $\mathbf{0}$ & $\mathbf{0}$ & $\mathbf{0}$ \\
\hline & Filtered & 0.5 & $\mathbf{0}$ & $\mathbf{0}$ & $\mathbf{0}$ & 0 & $\mathbf{0}$ \\
\hline & & $0 \cdot 2$ & $\mathbf{0}$ & $\mathbf{0}$ & $\mathbf{0}$ & $\mathbf{0}$ & $\mathbf{0}$ \\
\hline \multirow{4}{*}{$\begin{array}{l}\text { Bacteria washed and } \\
\text { penicillinase added }\end{array}$} & Not filtered & $0 \cdot 2$ & $\mathbf{2}$ & $\mathbf{2}$ & 2 & $\mathbf{2}$ & $\mathbf{2}$ \\
\hline & & $0 \cdot 1$ & $\mathbf{1}$ & $\mathbf{2}$ & 2 & 2 & $\mathbf{1}$ \\
\hline & Filtered & 0.5 & $\mathbf{0}$ & 2 & $\mathbf{2}$ & $\mathbf{2}$ & 0 \\
\hline & & 0.2 & 0 & 2 & 1 & $\mathbf{2}$ & $\mathbf{0}$ \\
\hline
\end{tabular}

$\mathrm{A}=S$. typhi $; \mathrm{B}=P$. vulgaris $; \mathrm{C}=$ P. morganii $; \mathrm{D}=$ E. coli $; \mathrm{E}=\boldsymbol{B}$. abortus (strain 19).

\section{Reactivation of the desulphhydrase activity by bacterial extracts}

Two bacterial extracts, using McIlwain's (1948) method, were prepared from Proteus vulgaris and Brucella abortus (strain 19). The respective protein content of the extracts was 6.8 and $5.6 \mathrm{mg} . / \mathrm{ml}$. When varying quantities of each extract were brought in contact with cysteine or sodium thiosulphate, hydrogen sulphide was produced as follows:

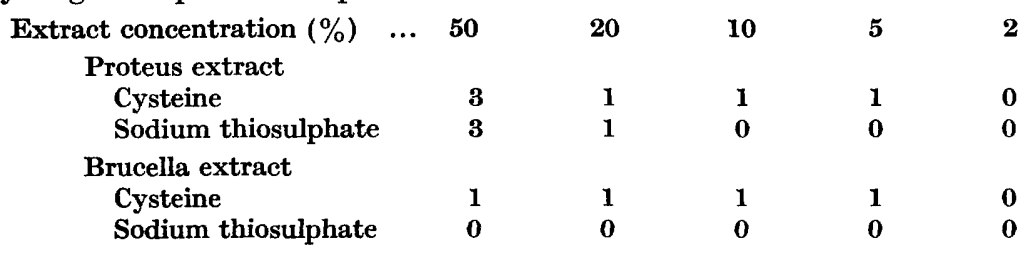


In order to avoid error from this source of hydrogen sulphide production, the extracts were added to the inactivated and washed bacteria in $1 \%$ concentrations on cysteine, and $10-20 \%$ on sodium thiosulphate.

As in the earlier experiments bacterial suspensions containing $10^{11}$ organisms/ $\mathrm{ml}$. were left in contact at $37^{\circ}$ with 25,000 units penicillin $/ \mathrm{ml}$. for $24 \mathrm{hr}$. The bacteria were then centrifuged, washed and resuspended in the substrate + buffer solutions, and bacterial extracts were added.

The low concentrations of extract only caused a slight reactivation of the hydrogen sulphide production by penicillin-inactivated bacteria (Table 13). This desulphhydrase activity was eliminated by heating extracts at $50^{\circ}$ for $\mathbf{3 0} \mathrm{min}$. while their reactivating power on inactivated bacteria was still intact. $10 \mu \mathrm{g} . / \mathrm{ml}$. of pyridoxal phosphate also exerted a slight reactivating activity.

Table 13. The reactivation of penicillin-inactivated suspensions Suspensions $10^{11} / \mathrm{ml}$. inactivated by $25,000 \mathrm{u}$. $/ \mathrm{ml}$.

\begin{tabular}{|c|c|c|c|c|c|c|}
\hline \multirow{2}{*}{$\begin{array}{l}\text { Extracts } \\
\text { from }\end{array}$} & \multirow{2}{*}{$\begin{array}{c}\text { Concentra- } \\
\text { tion } \\
(\%)\end{array}$} & \multirow{2}{*}{$\begin{array}{c}\text { Substrate } \\
\text { tested }\end{array}$} & \multicolumn{4}{|c|}{ Relative $\mathrm{H}_{2} \mathrm{~S}$ production by strains } \\
\hline & & & $\mathbf{A}$ & $\mathbf{B}$ & C & $\mathbf{D}$ \\
\hline B. abortus & $\begin{array}{r}1 \cdot 0 \\
20 \cdot 0\end{array}$ & $\begin{array}{l}\text { Cy. } \\
\text { T. }\end{array}$ & $\begin{array}{l}\mathbf{0} \\
\mathbf{0}\end{array}$ & $\begin{array}{l}0 \\
0\end{array}$ & $\begin{array}{l}\mathbf{0} \\
1\end{array}$ & $\begin{array}{l}\mathbf{1} \\
\mathbf{3}\end{array}$ \\
\hline P. vulgaris & $\begin{array}{c}1.0 \\
10 \cdot 0 \\
\text { Controls } \\
\text { without } \\
\text { extract }\end{array}$ & $\begin{array}{l}\text { Cy. } \\
\text { T. } \\
\text { Cy. } \\
\text { T. }\end{array}$ & $\begin{array}{l}0 \\
1 \\
0 \\
0\end{array}$ & $\begin{array}{l}\mathbf{0} \\
\mathbf{0} \\
\mathbf{0} \\
\mathbf{0}\end{array}$ & $\begin{array}{l}\mathbf{1} \\
\mathbf{1} \\
\mathbf{0} \\
\mathbf{0}\end{array}$ & $\begin{array}{l}\mathbf{1} \\
\mathbf{1} \\
\mathbf{0} \\
\mathbf{0}\end{array}$ \\
\hline
\end{tabular}

$\mathrm{A}=\boldsymbol{P}$. vulgaris $; \mathrm{B}=\boldsymbol{P}$. morganii $; \mathrm{C}=\boldsymbol{E}$. coli $; \mathrm{D}=\mathrm{B}$. abortus (strain 19 ). $\mathrm{Cy} .=$ cysteine; T. = Sodium thiosulphate.

These two activating factors, extracts heated at $50^{\circ}$ for $30 \mathrm{~min}$. and pyridoxal phosphate, were employed in the following experiments. Since the desulphhydrase activity of the extract was inactivated, $20 \%$ concentrations were used for the reactivation of the bacteria. The bacterial inocula and the concentration of penicillin employed for inactivation were the same as in the experiment shown in Table 13. Table 14 summarizes an experiment of this type. Pyridoxal phosphate exerted only a slight reactivating effect on the bacteria tested on cysteine. In comparison with this weak action, the reactivation by the bacterial extract was much stronger and in the presence of both extract and pyridoxal phosphate, hydrogen sulphide production was accelerated.

The next experiment was carried out with Proteus vulgaris. Extract was prepared from Brucella abortus and was heated at $50^{\circ}$ for $1 \mathrm{hr}$. The reactivation was tested on cysteine and different inocula and different concentrations of the two reactivating factors were employed. The inactivation of the bacteria was carried out as in the earlier experiments.

A control showed that the extract and pyridoxal phosphate either separately or together did not produce hydrogen sulphide on cysteine + buffer solution without the inactivated bacteria. The experiment summarized in Table 15 showed that inocula of $10^{11}$ and $10^{10}$ organisms $/ \mathrm{ml}$. were reactivated by 
2.5-20 $\mu \mathrm{g}$./ml. of pyridoxal phosphate, while this effect was not produced with an inoculum of $10^{9}$ organisms $/ \mathrm{ml}$.

Table 14. The reactivating effect of heated proteus extract and of pyridoxal phosphate

Suspensions $10^{11} / \mathrm{ml}$. inactivated by $25,000 \mathrm{u}$. penicillin $/ \mathrm{ml}$.

Quantities added

\begin{tabular}{|c|c|c|c|c|c|c|}
\hline \multirow{2}{*}{$\begin{array}{c}\text { Heated } \\
P . \text { vulgaris } \\
\text { extract } \\
(\%)\end{array}$} & \multirow{2}{*}{$\begin{array}{c}\text { Pyridoxal } \\
\text { phosphate } \\
(\mu \mathrm{g} \cdot / \mathrm{ml} .)\end{array}$} & \multirow{2}{*}{$\begin{array}{c}\text { Substrate } \\
\text { tested }\end{array}$} & \multicolumn{4}{|c|}{ Relative $\mathrm{H}_{2} \mathrm{~S}$ production by strain } \\
\hline & & & $\mathbf{A}$ & B & C & D \\
\hline 20 & $\mathbf{0}$ & Cy. & $\mathbf{3}$ & 3 & 3 & 2 \\
\hline 20 & 10 & Cy. & 3 & 3 & $\mathbf{3}$ & $\mathbf{3}$ \\
\hline 20 & o & T. & 2 & 2 & 2 & $\mathbf{0}$ \\
\hline 20 & 10 & T. & 3 & $\mathbf{3}$ & 2 & $\mathbf{0}$ \\
\hline 0 & 0 & Cy. & 0 & 0 & 0 & $\mathbf{0}$ \\
\hline 0 & 10 & Cy. & 1 & 1 & 0 & $\mathbf{0}$ \\
\hline 0 & 0 & T. & 0 & 0 & 0 & 0 \\
\hline 0 & 10 & T. & 0 & 0 & 0 & $\mathbf{0}$ \\
\hline
\end{tabular}

Strains A-D: the same as in Table 13. Cy.=cysteine; T.=sodium thiosulphate.

Table 15. Reactivation of inactivated $\mathbf{P}$. vulgaris suspensions by pyridoxal phosphate and B. abortus extract

\begin{tabular}{|c|c|c|c|c|c|c|}
\hline \multirow{3}{*}{$\begin{array}{c}\text { Concentrations } \\
\text { of the brucella } \\
\text { extract } \\
(\%)\end{array}$} & \multicolumn{6}{|c|}{ Proteus vulgaris suspension (cells/ml.) } \\
\hline & \multicolumn{2}{|c|}{$10^{11}$} & \multicolumn{2}{|c|}{$10^{10}$} & \multicolumn{2}{|c|}{$10^{9}$} \\
\hline & \multicolumn{6}{|c|}{ Relative $\mathbf{H}_{\mathbf{2}} \mathbf{S}$ production observed } \\
\hline 20 & $\mathbf{3}$ & 2 & $\mathbf{3}$ & 2 & $\mathbf{3}$ & $\mathbf{3}$ \\
\hline 10 & $\mathbf{3}$ & 2 & 2 & 2 & $\mathbf{3}$ & 2 \\
\hline 5 & 2 & 1 & 2 & 1 & 2 & 1 \\
\hline 2 & 2 & 1 & 2 & 1 & 2 & $\mathbf{1}$ \\
\hline 1 & 2 & $\mathbf{0}$ & 2 & $\mathbf{0}$ & 2 & $\mathbf{1}$ \\
\hline $\begin{array}{l}\text { Control with- } \\
\text { out extract }\end{array}$ & $\mathbf{2}$ & $\mathbf{0}$ & $\mathbf{2}$ & $\mathbf{0}$ & 0 & $\mathbf{0}$ \\
\hline
\end{tabular}

P. $=2 \cdot 5-20 \mu \mathrm{g} . / \mathrm{ml}$. pyridoxal phosphate added $;$ NP. $=$ pyridoxal phosphate not added.

In higher concentrations of the bacterial extract and in the absence of pyridoxal phosphate, all inocula produced hydrogen sulphide within $24 \mathrm{hr}$., while the lower concentrations were relatively inactive and did not exert much effect. It seems, therefore, that for a complete reactivation of small inocula of Proteus vulgaris $\left(10^{9}\right.$ organisms $/ \mathrm{ml}$.) two different methods may be employed: high concentrations of bacterial extracts or low concentrations of bacterial extracts simultaneously with pyridoxal phosphate. However, the reactivation of suspensions containing $10^{10}-10^{11}$ organisms $/ \mathrm{ml}$. can also be effected by pyridoxal phosphate alone.

Suspensions containing $10^{11}$ organisms $/ \mathrm{ml}$. were kept in the ice-box for 14. days to autolyse, then heated at $100^{\circ}$ for $1 \mathrm{hr}$., and centrifuged until the supernatant fluid became completely clear. $50 \%$ concentrations of these 
extracts exerted some reactivating effects. An extract prepared from Brucella abortus reactivated Proteus morganii and Escherichia coli on cysteine and sodium thiosulphate, but failed to reactivate Salmonella typhi and Brucella abortus. While these reactivating effects were observed, the extracted bacterial suspensions failed to exert such an effect.

\section{REFERENCES}

BeiJerinck, M. W. (1901). Sur la formation de l'hydrogène sulfuré dans les canaux et le genre nouveau Aërobacter. Arch. néerl. Sci. (Ser. 2), 4, 1.

Beijerinck, M. W. (1904). Phénomènes de reduction produits par les microbes. Arch. néerl. Sci. (Ser. 2), 9, 131.

Braun, H. \& Silberstein, W. (1942). Über die Bedingungen der Schwefelwasserstoff-bildung by Darmbakterien. Rev. Fac. Sci. Univ. Istanbul, B, 7, 1.

Clarke, P. H. (1953). Hydrogen sulphide production by bacteria. J.gen. Microbiol. $8,397$.

DeLwiche, A. (1951). Activators for the cysteine desulfhydrase system of Escherichia coli mutant. J. Bast. 62, 717.

KaLLIO, R. E. (1951). Function of pyridoxal phosphate in desulfhydrase systems of Proteus morganii. J. biol. Chem. 192, 371.

Kallio, R. E. \& Porter, J. R. (1950). The metabolism of cystine and cysteine by Proteus vulgaris and Proteus morganii. J. Bact. 60, 607.

McIlwain, H. (1948). Preparation of cell-free extracts with powdered alumina. J. gen. Microbiol. $2,288$.

ÖzEK, Ö. (1951). Die Indol und Schwefelwasserstoff bildung der Salmonellen und Shigellen unter verschiedenen Ernährungsbedingungen. $Z$. Bakt. (I. Orig.), $157,56$.

Seligmand, E. \& Wassermann, M. (1947). Induced resistance to streptomycin. J. Immunol. 57, 351.

Skariton-Loewenthal, M., Rappaport, F. A. \& Olitzki, A. L. (1953). Inhibitory action of some inorganic compounds and antibiotics on the $\mathrm{H}_{2} \mathrm{~S}$ production of Salmonellae. Bull. Res. Counc. Israel, 2, 444.

(Received 10 February 1954) 